OPEN ACCESS

Edited by:

Antonio Cappuccio,

Mount Sinai Hospital, United States

Reviewed by:

Michael Platten,

Karolinska Institutet (KI), Sweden

Mostafa Salem,

Assiut University, Egypt

*Correspondence:

Faezeh Moazam

faezeh.moazami@inserm.fr

Specialty section:

This article was submitted to Inflammation,

a section of the journal

Frontiers in Immunology

Received: 28 April 2021

Accepted: 26 July 2021

Published: 11 August 2021

Citation:

Moazami F, Lefevre-Utile A,

Papaloukas C and Soumelis V (2021) Machine Learning Approaches in

Study of Multiple Sclerosis

Disease Through Magnetic Resonance Images.

Front. Immunol. 12:700582. doi: 10.3389/fimmu.2021.700582

\section{Machine Learning Approaches in Study of Multiple Sclerosis Disease Through Magnetic Resonance Images}

\author{
Faezeh Moazami ${ }^{1 *}$, Alain Lefevre-Utile ${ }^{1,2,3}$, Costas Papaloukas ${ }^{4}$ and Vassili Soumelis ${ }^{1,5}$ \\ 1 Université de Paris, Institut de Recherche Saint-Louis, Institut National de la Santé et de la Recherche Médicale (INSERM) \\ U976, Hôpital Saint-Louis, Paris, France, ${ }^{2}$ Université Paris-Saclay, Saint Aubin, France, ${ }^{3}$ Assistance Publique Hopitaux de \\ Paris (APHP), General Pediatric and Pediatric Emergency Department, Jean Verdier Hospital, Bondy, France, ${ }^{4}$ Department \\ of Biological Applications and Technology, University of loannina, Ioannina, Greece, ${ }^{5}$ Assistance Publique Hopitaux de Paris \\ (APHP), Hôpital Saint-Louis, Immunology-Histocompatibility Department, Paris, France
}

Multiple sclerosis (MS) is one of the most common autoimmune diseases which is commonly diagnosed and monitored using magnetic resonance imaging (MRI) with a combination of clinical manifestations. The purpose of this review is to highlight the main applications of Machine Learning (ML) models and their performance in the MS field using MRI. We reviewed the articles of the last decade and grouped them based on the applications of ML in MS using MRI data into four categories: 1) Automated diagnosis of MS, 2) Prediction of MS disease progression, 3) Differentiation of MS stages, 4) Differentiation of MS from similar disorders.

Keywords: artificial intelligence, machine learning, multiple sclerosis, disability prediction, magnetic resonance imaging (MRI)

\section{INTRODUCTION}

Multiple sclerosis (MS) is one of the main causes of acquired neurologic disability in young adults. Its prevalence varies from 5 to 300 per 100000 (1) representing 2 to 3 million people globally (2). Disease evolution is marked by unpredictable flares of autoimmune and inflammatory central nervous system demyelination and axonal transection (3). According to the disease course, different MS stages exist. Relapsing Remitting MS (RRMS) is the most common form of MS. More than $80 \%$ will experience RRMS with neurological exacerbations separated by complete or incomplete remission (4). Secondary progressive MS (SPMS) develops from RRMS, followed by gradual neurologic deterioration not associated with acute attacks (5). Few patients will evolve into a Primary-Progressive MS (PPMS) or Progressive-Relapsing MS (PRMS) with gradual deterioration without recovery (6). Clinical presentation and detection of damage to the nervous system could help to study multiple sclerosis (MS). As MS early stage can be underdiagnosed due to non-specific clinical presentation, MRI (Magnetic Resonance Imaging) is crucial to diagnose, estimate the disease stage and predict the outcome (7). Brain lesions in MR images are an efficient imaging biomarker for multiple sclerosis diagnosis. Since detection of these lesions is laborious and time consuming and 
depends on radiologist experience, image processing methods based on object classification using ML learning techniques are used to apply automatic segmentation on MR images.

\section{METHOD}

\section{Search Strategy}

We conducted an electronic search on PubMed in March 2021 for the studies published from January 1st, 2011 to March 31st, 2021. The articles were searched with several appropriate keywords in combination with the Boolean operators:

Multiple sclerosis AND [ML Learning OR Artificial intelligence OR Deep learning OR Neural network] AND Magnetic resonance.

\section{Inclusion Criteria}

We selected original publications written in English.

\section{Exclusion Criteria}

Duplicate studies, reviews, comparative publications and case reports were excluded. Any studies not using methods of ML learning were also excluded.
In total, 106 studies were obtained that all used ML Learning models to investigate MS disease through MR images. From those, 52 were omitted according to the exclusion criteria.

\section{RESULTS}

After the review process of the 54 publications, we sectioned the studies into the following four categories: 1) Automated diagnosis of MS, 2) Prediction of disease progression, 3) Differentiation of MS stages, 4) Differentiation of MS disease from similar disorders.

Figure 1 shows the steps of applying ML Learning classifiers on MRI images to study MS disease for the four different tasks.

We observed that the input MRI images contained different types of data including images of 1) MS patients and a healthy control group, 2) MS patients and a set of images that were segmented by an expert and considered as ground truth, 3) Patients with different stages of MS, 4) patients with MS and other diseases, very similar to MS. The images then were analyzed to extract the most important biomarkers such as whole brain atrophy biomarkers, gray matter atrophy biomarkers, spinal cord atrophy biomarkers, etc. The detection process of these lesions was carried out by using different image

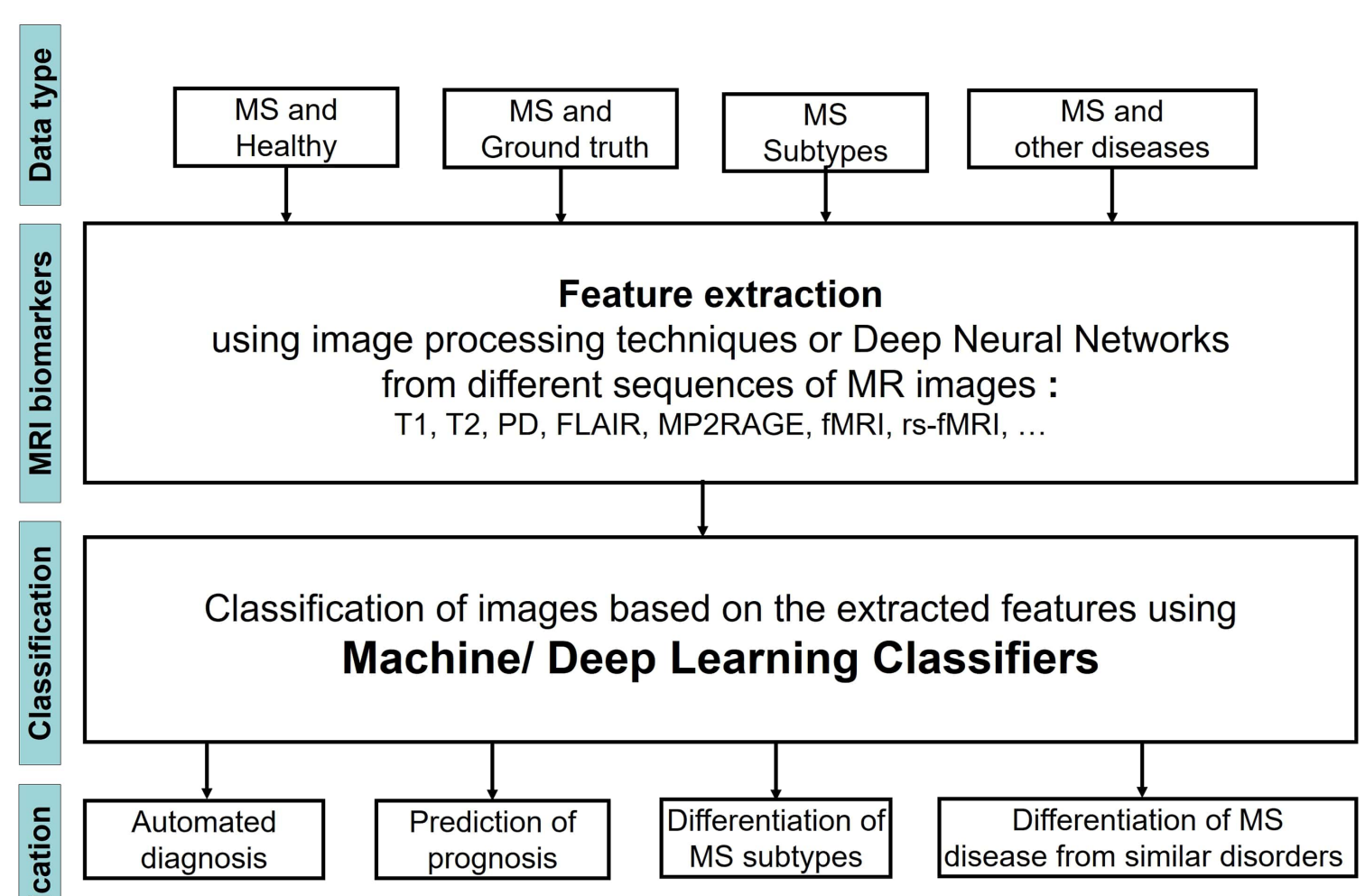

FIGURE 1 | Flowchart showing the process of using ML Learning to study MS through MRI images. 
processing methods that are based on thresholds or various models of Deep Learning (DL). Afterwards a classifier was applied on the extracted features of the previous step to classify the MRI images into several groups and reach the aim of the study that could be one or two of the four categories: 1) Automated diagnosis of MS, 2) Prediction of MS disease progression, 3) Differentiation of MS stages, 4) Differentiation of MS from similar disorders. The classifier could be a typical ML learning model (i.e. Support Vector Machine, Random Forest, etc.) or the layer in a DL model.

The performance of both segmentation and classification steps were also quantified with various evaluation metrics. In this review, we use Accuracy (ACC), Sensitivity, Dice Similarity Coefficient (DSC), and Area Under the Curve (AUC), Root Mean Square Error (RMSE), to present performance of the models. These metrics are derived from the confusion matrix which is a two by two table formed by calculating the True Positive classified objects (TP), True Negative classified objects (TN), False Positive classified objects (FP), and False Negative classified objects (FN).

$$
\begin{gathered}
A C C=\frac{T P+T N}{T P+T N+F N+F P} \\
\text { Sensitivity }=\frac{T P}{T P+F N} \\
\text { DSC }=\frac{2 T P}{2 T P+F N+F P} \\
\text { RMSE }=\sqrt{\frac{1}{N} \sum_{i=1}^{N}(\hat{y} i-y i)^{2}}
\end{gathered}
$$

Where $\mathrm{N}=$ total number of values, $\hat{\mathrm{y}}=$ predicted values, $\mathrm{y}=$ actual values.

AUC also measures the entire two-dimensional area underneath the entire ROC curve which plots two parameters of True Positive Rate and False Positive Rate (FPR).

In Table 1 the summary information of the studies is described, including the quantification of the publications, the employed ML learning models and their best performances, the MRI sequences used as input images for feature extraction and the number of patients in the studies.

Most of the articles studied here, have focused on automated diagnosis of MS whereas only two publications have used ML models to differentiate MRI images of MS stages. Three different strategies were used in the studies for applying ML learning models on the images. In the first strategy the lesions in the images were segmented using image processing methods, then the extracted texture features were fed to a typical ML learning model to classify the images. The second strategy used a deep Neural Network for both feature extraction from the MR images and classification of the images based on the obtained features. We also found two articles that used a combination of the first two strategies. Specifically, the first layers of a DL model extracted the features from the images, while a standard ML learning classifier was applied on the feature map obtained from the DL model.

Since the most widely used models are Neural Networks, we divided the different types of neural network into several groups to better apprehend their performances: Deep Neural Networks without pre-training, Deep Neural Networks with pre-training (TL method), U-net and Other NNs.

\section{Automated Diagnosis of MS}

According to the McDonald and CSF criteria, the diagnosis process of MS is based on clinical presentation as well as brain and spinal cord MRI to study the dissemination of central nervous system (CNS) lesions in time and space (55). In recent years, a range of studies have proposed ML learning methods for automatic detection of central nervous system lesions from MRI on patients with MS. One of the best results for detecting T1-w and T2-w MS lesions we found in the articles, was obtained from a CNN with an accuracy of $98.8 \%$. This model was proposed by Rocca et al. (18) and is based on four three-dimensional convolutional layers, followed by a fully dense layer after the feature extraction. It was trained with 178 scans from 268 patients.

The common problem of using CNNs is that tuning a huge number of parameters and initialize the weights are both very complex processes. In addition, such networks require a large amount of data to generalize and perform well. TL can solve these problems by employing pre-trained models, trained on a large dataset. Therefore some recent studies tried to improve DL model performance using this method. For instance Eitel et al. (8) investigated the performance of $3 \mathrm{D}$ convolutional neural networks (CNNs) and layer-wise relevance propagation (LRP) for the detection of brain lesions in MRI images of MS patients $(n=76)$ and healthy controls $(n=71)$. They used a network that was already pre-trained on a MRI dataset from 921 patients with Alzheimer's disease. Subsequently the pre-trained model was applied on the MS data to classify them into two groups, disease and healthy. The proposed models reached an accuracy level of 87.04\% and an AUC of 0.96 .

Another common DL architecture in MS image analysis is the U-net, which was developed by Ronneberger et al. (9). This model contains a fully convolutional neural network (FCNN) that includes contraction and expanding paths to perform segmentation. We found six studies that report high performance levels and applied this model on different sequences of MRI, as shown in the Table $\mathbf{1}$.

Furthermore, the most common typical ML model used in all studies is SVM. For instance, Zurita et al. (56) extracted fractional anisotropy maps, structural and functional connectivity from MR images as biomarkers to diagnose patients with relapsing-remitting multiple sclerosis and healthy volunteers. Then they applied SVM on the extracted features, which was able to distinguish the two groups of patients and the healthy controls with a high level of accuracy $(89 \% \pm 2 \%)$. 
TABLE 1 | 54 publications were grouped into 4 categories based on the application of ML Learning in MS disease.

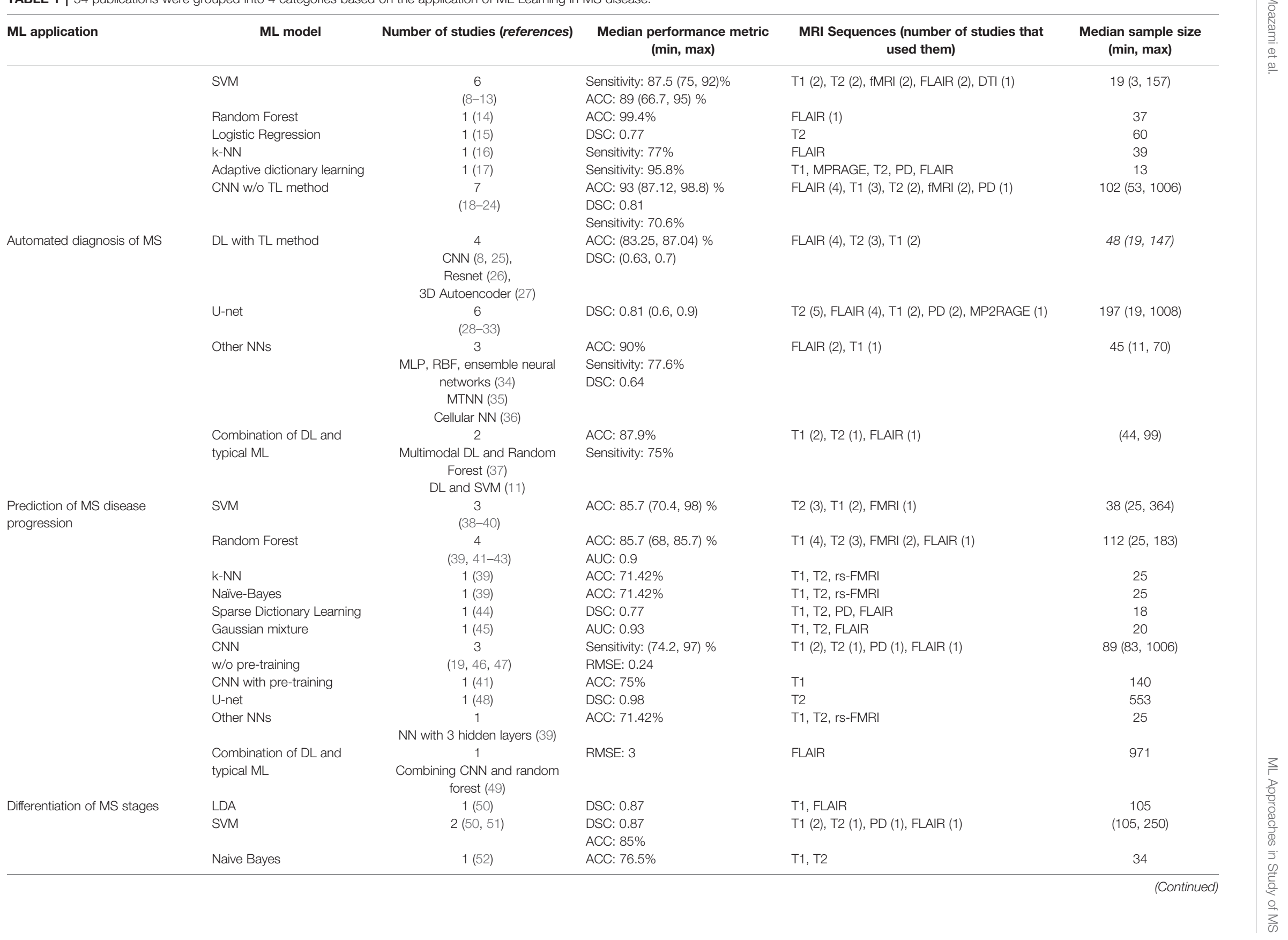




\section{Prediction of MS Disease Progression}

One of the challenges in MS evaluation and the prediction of its stages is that the symptoms vary widely and as the disease worsens, new lesions become less frequent (57). In addition, the MS course in patients, even from the early stages, is characterized by a slow progression of disabilities independent of relapses (58). Automatic segmentation of MS lesions, including gadolinium-enhancing, new T2 or enlarging T2, are essential biomarkers for the progression of the disease as we as the treatment options and allow to explore the morphological changes in relation to clinical disease burden $(48,59)$.

Our review shows that three studies in this section applied ML to predict the progression of MS by measuring Expanded Disability Status Scale (EDSS) in the first years of disease evolution $(38,46,49)$. Two of them used CNN for feature extraction and image classification, and the third one applied SVM for MR image classification based on the extracted features from the images. And also as shown in Table 1, SVM and Random Forests are the most commonly used models for studying the prognosis of MS patients, while both provide a high level of performance.

One of the articles in this direction assessed the influence of lesion volumes on the $\mathrm{CNN}$ detection performance. Coronado et al. (19) applied this model on five multispectral MRI images with different volumes of gadolinium-enhancing. They obtained the best performance by utilizing as input all five multispectral image sets, including FLAIR, T2, PD, and pre- and post-contrast T1, as well as when the enhancement size of the lesions was increased.

Youngjin Yoo et al. (41) improved the performance of their $\mathrm{CNN}$ model in predicting the lesions progression by using a TL method. At first they applied an unsupervised 3D convolutional deep belief network (DBN) as a pre-trained model, and then a CNN was used to extract latent MS lesion patterns. They also compared their model against the performance of Random Forests. Their CNN model resulted in an average accuracy of $75.0 \%$ in predicting the clinical conversion to definite MS within two years, while the Random Forests yielded an accuracy of $67.9 \%$.

\section{Differentiation of MS Stages}

In our review, we found two studies that differentiated the stages of MS patients using biomarkers such as lesion location and metabolic features and SVM classifier. Specifically, IonMărgineanu et al. (50) combined lesion loads (total amount of lesion area) with clinical data magnetic resonance metabolic features to classify 87 MS patients into four groups based on the progression stages. They applied two different classification models on the extracted features: LDA and SVM with a Gaussian kernel (SVM-rbf). The highest classification (F1score: 87\%) was obtained for RRMS and SPMS after training the LDA and SVM-rbf models on clinical, lesion loads and metabolic features.

\section{Differentiation of MS Disease From Similar Disorders}

Another important step in the diagnosis of MS is the differentiation of the disease from other similar disorders, since, despite their similarities, the treatments are greatly 
different. In our search we retrieved three publications related to this objective.

Two of them used different biomarkers and ML learning classifiers to distinguish MS from Neuromyelitis Optical Spectrum Disorders (NMOSD) $(53,54)$. The first article employed a CNN for lesion segmentation of MRI images on 213 patients with MS and 125 patients with NMOSD. This model yielded an accuracy of $71.1 \%$ in differentiating NMOSD from MS images. The second study utilized TL to pre-train two different architectures of CNN model on the ImageNet dataset (60); one model with 34 layers and one with 18 layers. The achieved accuracies of the two models were 0.75 and 0.725 , respectively. In the last study Mato-Abad et al. (52) exploited a Naive Bayes classifier in order to distinguish two types of MS patients: Radiologically Isolated Syndrome (RIS) and CIS, and obtained an accuracy of 0.765 , based on the morphometric measurements in MRI images.

\section{CONCLUSION}

In this review we differentiated the applications of ML learning in the field of MS disease. The results show that ML can reliably support the efforts in the research field of MS disease. However, it is not possible to directly compare all the methods proposed in the related literature, since most of the works use different performance metrics to evaluate their results while the lesion volumes that were segmented in the MR images vary greatly among the studies. Nevertheless, in all ML applications, one of

\section{REFERENCES}

1. Browne P, Chandraratna D, Angood C, Tremlett H, Baker C, Taylor BV, et al. Atlas of Multiple Sclerosis 2013: A Growing Global Problem With Widespread Inequity. Neurology (2014) 83(11):1022-4. doi: 10.1212/ WNL.0000000000000768

2. Wallin MT, Culpepper WJ, Campbell JD, Nelson LM, Langer-Gould A, Marrie RA, et al. The Prevalence of MS in the United States: A PopulationBased Estimate Using Health Claims Data. Neurology (2019) 92(10):e102940. doi: 10.1212/WNL.0000000000007035

3. Thompson AJ, Baranzini SE, Geurts J, Hemmer B, Ciccarelli O. Multiple Sclerosis. Lancet (2018) 391(10130):1622-36. doi: 10.1016/S0140-6736(18)30481-1

4. Lublin FD, Reingold SC. Defining the Clinical Course of Multiple Sclerosis: Results of an International Survey. National Multiple Sclerosis Society (USA) Advisory Committee on Clinical Trials of New Agents in Multiple Sclerosis. Neurology (1996) 46(4):907-11. doi: 10.1212/wnl.46.4.907

5. Koch M, Kingwell E, Rieckmann P, Tremlett H, MS Clinic Neurologists UBC. The Natural History of Secondary Progressive Multiple Sclerosis. J Neurol Neurosurg Psychiatry (2010) 81(9):1039-43. doi: 10.1136/jnnp.2010.208173

6. Faissner S, Plemel JR, Gold R, Yong VW. Progressive Multiple Sclerosis: From Pathophysiology to Therapeutic Strategies. Nat Rev Drug Discovery (2019) 18 (12):905-22. doi: 10.1038/s41573-019-0035-2

7. McGinley MP, Goldschmidt CH, Rae-Grant AD. Diagnosis and Treatment of Multiple Sclerosis: A Review. JAMA (2021) 325(8):765-79. doi: 10.1001/ jama.2020.26858

8. Eitel F, Soehler E, Bellmann-Strobl J, Brandt AU, Ruprecht K, Giess RM, et al. Uncovering Convolutional Neural Network Decisions for Diagnosing Multiple Sclerosis on Conventional MRI Using Layer-Wise Relevance Propagation. NeuroImage Clin (2019) 24:102003. doi: 10.1016/j.nicl.2019.102003 the most widely used models are the deep neural networks, a fact that indicates the significance of these models in the field of MS studies over the last decade. Feature extraction and selection from MR images by an expert does not allow for finding new and hidden information in the data. DL models could help to overcome this issue by extracting useful information directly from raw image data. On the other hand, the utilization of TL methods during the training process of DL models, could help to increase their performance. This approach has shown notable performance, especially in medical research where usually limited image datasets are available while image annotation by an expert is a rather tedious task.

\section{AUTHOR CONTRIBUTIONS}

FM, AL-U, and CP designed the project. FM studied the selected publications and wrote the manuscript. AL-U approved the medical concepts of the manuscript. CP and VS directed the project. All authors contributed to the article and approved the submitted version.

\section{FUNDING}

FM was supported by the ANR DENDRITISEPSIS (ANR-17CE15-003), and A.L-U by the Fondation pour la Recherche Médicale (FDM201806006187).
9. Ronneberger O, Fischer P, Brox T. U-Net: Convolutional Networks for Biomedical Image Segmentation. In: N Navab, J Hornegger, W Wells, A Frangi, editors. Medical Image Computing and Computer-Assisted Intervention - MICCAI 2015. MICCAI 2015. Lecture Notes in Computer Science, vol. 9351. Springer, Cham (2015). doi: 10.1007/978-3-319-24574$4 \_28$

10. Azarmi F, Miri Ashtiani SN, Shalbaf A, Behnam H, Daliri MR. Granger Causality Analysis in Combination With Directed Network Measures for Classification of MS Patients and Healthy Controls Using Task-Related fMRI. Comput Biol Med (2019) 115:103495. doi: 10.1016/j.compbiomed.2019.103495

11. Kuwazuru J, Arimura H, Kakeda S, Yamamoto D, Magome T, Yamashita Y, et al. Automated Detection of Multiple Sclerosis Candidate Regions in MR Images: False-Positive Removal With Use of an ANN-Controlled Level-Set Method. Radiol Phys Technol (2012) 5(1):105-13. doi: 10.1007/s12194-0110141-2

12. Reitz SC, Hof SM, Fleischer V, Brodski A, Gröger A, Gracien RM, et al. MultiParametric Quantitative MRI of Normal Appearing White Matter in Multiple Sclerosis, and the Effect of Disease Activity on T2. Brain Imaging Behav (2017) 11(3):744-53. doi: 10.1007/s11682-016-9550-5

13. Bendfeldt K, Klöppel S, Nichols TE, Smieskova R, Kuster P, Traud S, et al. Multivariate Pattern Classification of Gray Matter Pathology in Multiple Sclerosis. Neuroimage (2012) 60(1):400-8. doi: 10.1016/j.neuroimage.2011.12.070

14. Melingi SB, Vijayalakshmi V. A Hybrid Approach for Sub-Acute Ischemic Stroke Lesion Segmentation Using Random Decision Forest and Gravitational Search Algorithm. Curr Med Imaging Rev (2019) 15(2):170-83. doi: 10.2174/ 1573405614666180209150338

15. Salem M, Cabezas M, Valverde S, Pareto D, Oliver A, Salvi J, et al. A Supervised Framework With Intensity Subtraction and Deformation Field Features for the Detection of New T2-W Lesions in Multiple Sclerosis. NeuroImage Clin (2017) 17:607-15. doi: 10.1016/j.nicl.2017.11.015 
16. Fartaria MJ, Bonnier G, Roche A, Kober T, Meuli R, Rotzinger D, et al. Automated Detection of White Matter and Cortical Lesions in Early Stages of Multiple Sclerosis. J Magn Reson Imaging (2016) 43(6):1445-54. doi: 10.1002/jmri.25095

17. Deshpande H, Maurel P, Barillot C. Classification of Multiple Sclerosis Lesions Using Adaptive Dictionary Learning. Comput Med Imaging Graph (2015) 46 Pt 1:2-10. doi: 10.1016/j.compmedimag.2015.05.003

18. Rocca MA, Anzalone N, Storelli L, Del Poggio A, Cacciaguerra L, Manfredi AA, et al. Deep Learning on Conventional Magnetic Resonance Imaging Improves the Diagnosis of Multiple Sclerosis Mimics. Invest Radiol (2021) Apr 156(4):252-60. doi: 10.1097/RLI.0000000000000735

19. Coronado I, Gabr RE, Narayana PA. Deep Learning Segmentation of Gadolinium-Enhancing Lesions in Multiple Sclerosis. Mult Scler (2021) 27 (4):519-27. doi: 10.1177/1352458520921364

20. Maggi P, Fartaria MJ, Jorge J, La Rosa F, Absinta M, Sati P, et al. CVSnet: A Machine Learning Approach for Automated Central Vein Sign Assessment in Multiple Sclerosis. NMR Biomed (2020) 33(5):e4283. doi: 10.1002/nbm.4283

21. Lopatina A, Ropele S, Sibgatulin R, Reichenbach JR, Güllmar D. Investigation of Deep-Learning-Driven Identification of Multiple Sclerosis Patients Based on Susceptibility-Weighted Images Using Relevance Analysis. Front Neurosci (2020) 14:609468. doi: 10.3389/fnins.2020.609468

22. Barquero G, La Rosa F, Kebiri H, Lu PJ, Rahmanzadeh R, Weigel M, et al. RimNet: A Deep 3D Multimodal MRI Architecture for Paramagnetic Rim Lesion Assessment in Multiple Sclerosis. NeuroImage Clin (2020) 28:102412. doi: 10.1016/j.nicl.2020.102412

23. Ackaouy A, Courty N, Vallée E, Commowick O, Barillot C, Galassi F. Unsupervised Domain Adaptation With Optimal Transport in Multi-Site Segmentation of Multiple Sclerosis Lesions From MRI Data. Front Comput Neurosci (2020) 14:19. doi: 10.3389/fncom.2020.00019

24. Valverde S, Cabezas M, Roura E, González-Villà S, Pareto D, Vilanova JC, et al. Improving Automated Multiple Sclerosis Lesion Segmentation With a Cascaded 3D Convolutional Neural Network Approach. Neuroimage (2017) 155:159-68. doi: 10.1016/j.neuroimage.2017.04.034

25. Essa E, Aldesouky D, Hussein SE, Rashad MZ. Neuro-Fuzzy Patch-Wise RCNN for Multiple Sclerosis Segmentation. Med Biol Eng Comput (2020) 58 (9):2161-75. doi: 10.1007/s11517-020-02225-6

26. Aslani S, Dayan M, Storelli L, Filippi M, Murino V, Rocca MA, et al. Multi-Branch Convolutional Neural Network for Multiple Sclerosis Lesion Segmentation. Neuroimage (2019) 196:1-15. doi: 10.1016/j.neuroimage.2019.03.068

27. Brosch T, Tang LY, Yoo Y, Li DK, Traboulsee A, Tam R. Deep 3d Convolutional Encoder Networks With Shortcuts for Multiscale Feature Integration Applied to Multiple Sclerosis Lesion Segmentation. IEEE Trans Med Imaging (2016) 35(5):1229-39. doi: 10.1109/TMI.2016.2528821

28. Gabr RE, Coronado I, Robinson M, Sujit SJ, Datta S, Sun X, et al. Brain and Lesion Segmentation in Multiple Sclerosis Using Fully Convolutional Neural Networks: A Large-Scale Study. Mult Scler (2020) 26(10):1217-26. doi: $10.1177 / 1352458519856843$

29. Brugnara G, Isensee F, Neuberger U, Bonekamp D, Petersen J, Diem R, et al. Automated Volumetric Assessment With Artificial Neural Networks Might Enable a More Accurate Assessment of Disease Burden in Patients With Multiple Sclerosis. Eur Radiol (2020) 30(4):2356-64. doi: 10.1007/s00330-01906593-y

30. La Rosa F, Abdulkadir A, Fartaria MJ, Rahmanzadeh R, Lu PJ, Galbusera R, et al. Multiple Sclerosis Cortical and WM Lesion Segmentation at 3T MRI: A Deep Learning Method Based on FLAIR and MP2RAGE. NeuroImage Clin (2020) 27:102335. doi: 10.1016/j.nicl.2020.102335

31. Narayana PA, Coronado I, Sujit SJ, Sun X, Wolinsky JS, Gabr RE. Are MultiContrast Magnetic Resonance Images Necessary for Segmenting Multiple Sclerosis Brains? A Large Cohort Study Based on Deep Learning. Magn Reson Imaging (2020) 65:8-14. doi: 10.1016/j.mri.2019.10.003

32. Salem M, Valverde S, Cabezas M, Pareto D, Oliver A, Salvi J, et al. A Fully Convolutional Neural Network for New T2-W Lesion Detection in Multiple Sclerosis. NeuroImage Clin (2020) 25:102149. doi: 10.1016/j.nicl.2019.102149

33. Fenneteau A, Bourdon P, Helbert D, Fernandez-Maloigne C, Habas C, Guillevin R. Investigating Efficient CNN Architecture for Multiple Sclerosis Lesion Segmentation. J Med Imaging (Bellingham) (2021) 8(1):14504. doi: 10.1117/1.JMI.8.1.014504

34. Fooladi M, Sharini H, Masjoodi S, Khodamoradi E. A Novel Classification Method Using Effective Neural Network and Quantitative Magnetization
Transfer Imaging of Brain White Matter in Relapsing Remitting Multiple Sclerosis. J BioMed Phys Eng (2018) 8(4):409-22. doi: 10.31661/ jbpe.v8i4Dec.926

35. Khastavaneh H, Ebrahimpour-Komleh H. Neural Network-Based Learning Kernel for Automatic Segmentation of Multiple Sclerosis Lesions on Magnetic Resonance Images. J BioMed Phys Eng (2017) 7(2):155-62.

36. Cerasa A, Bilotta E, Augimeri A, Cherubini A, Pantano P, Zito G, et al. A Cellular Neural Network Methodology for the Automated Segmentation of Multiple Sclerosis Lesions. J Neurosci Methods (2012) 203(1):193-9. doi: 10.1016/j.jneumeth.2011.08.047

37. Yoo Y, Tang LYW, Brosch T, Li DKB, Kolind S, Vavasour I, et al. Deep Learning of Joint Myelin and T1w MRI Features in Normal-Appearing Brain Tissue to Distinguish Between Multiple Sclerosis Patients and Healthy Controls. NeuroImage Clin (2017) 17:169-78. doi: 10.1016/j.nicl.2017.10.015

38. Loizou CP, Pantzaris M, Pattichis CS. Normal Appearing Brain White Matter Changes in Relapsing Multiple Sclerosis: Texture Image and Classification Analysis in Serial MRI Scans. Magn Reson Imaging (2020) 73:192-202. doi: 10.1016/j.mri.2020.08.022

39. Saccà V, Sarica A, Novellino F, Barone S, Tallarico T, Filippelli E, et al. Evaluation of Machine Learning Algorithms Performance for the Prediction of Early Multiple Sclerosis From Resting-State FMRI Connectivity Data. Brain Imaging Behav (2019) 13(4):1103-14. doi: 10.1007/s11682-018-9926-9

40. Bendfeldt K, Taschler B, Gaetano L, Madoerin P, Kuster P, Mueller-Lenke N, et al. MRI-Based Prediction of Conversion From Clinically Isolated Syndrome to Clinically Definite Multiple Sclerosis Using SVM and Lesion Geometry. Brain Imaging Behav (2019) 13(5):1361-74. doi: 10.1007/s11682-018-9942-9

41. Yoo Y, Tang LYW, Li DKB, Metz L, Kolind S, Traboulsee AL, et al. Deep Learning of Brain Lesion Patterns and User-Defined Clinical and MRI Features for Predicting Conversion to Multiple Sclerosis From Clinically Isolated Syndrome. Comput Methods Biomech Biomed Eng: Imaging Visualization (2019) 7(3):250-9. doi: 10.1080/21681163.2017.1356750

42. Buyukturkoglu K, Zeng D, Bharadwaj S, Tozlu C, Mormina E, Igwe KC, et al. Classifying Multiple Sclerosis Patients on the Basis of SDMT Performance Using Machine Learning. Mult Scler (2021) 27(1):107-16. doi: 10.1177/ 1352458520958362

43. Zhang H, Alberts E, Pongratz V, Mühlau M, Zimmer C, Wiestler B, et al. Predicting Conversion From Clinically Isolated Syndrome to Multiple Sclerosis-An Imaging-Based Machine Learning Approach. NeuroImage Clin (2019) 21:101593. doi: 10.1016/j.nicl.2018.11.003

44. Roy S, He Q, Sweeney E, Carass A, Reich DS, Prince JL, et al. Subject-Specific Sparse Dictionary Learning for Atlas-Based Brain MRI Segmentation. IEEE $J$ BioMed Health Inform (2015) 19(5):1598-609. doi: 10.1109/JBHI.2015.2439242

45. Lesjak Ž, Pernuš F, Likar B, Špiclin Ž. Validation of White-Matter Lesion Change Detection Methods on a Novel Publicly Available MRI Image Database. Neuroinformatics (2016) 14(4):403-20. doi: 10.1007/s12021-0169301-1

46. Marzullo A, Kocevar G, Stamile C, Calimeri F, Terracina G, Durand-Dubief F, et al. Prediction of Multiple Sclerosis Patient Disability From Structural Connectivity Using Convolutional Neural Networks. Annu Int Conf IEEE Eng Med Biol Soc (2019) 2019:2087-90. doi: 10.1109/EMBC.2019.8856845

47. Gessert N, Krüger J, Opfer R, Ostwaldt AC, Manogaran P, Kitzler HH, et al. Multiple Sclerosis Lesion Activity Segmentation With Attention-Guided TwoPath CNNs. Comput Med Imaging Graph (2020) 84:101772. doi: 10.1016/ j.compmedimag.2020.101772

48. Platten M, Brusini I, Andersson O, Ouellette R, Piehl F, Wang C, et al. Deep Learning Corpus Callosum Segmentation as a Neurodegenerative Marker in Multiple Sclerosis. J Neuroimaging (2021) May31(3):493-500. doi: 10.1111/ jon. 12838

49. Roca P, Attye A, Colas L, Tucholka A, Rubini P, Cackowski S, et al. OFSEP Investigators; Steering Committee; Investigators; Imaging Group. Artificial Intelligence to Predict Clinical Disability in Patients With Multiple Sclerosis Using FLAIR MRI. Diagn Interv Imaging (2020) 101(12):795-802. doi: 10.1016/j.diii.2020.05.009

50. Ion-Mărgineanu A, Kocevar G, Stamile C, Sima DM, Durand-Dubief F, Van Huffel S, et al. Machine Learning Approach for Classifying Multiple Sclerosis Courses by Combining Clinical Data With Lesion Loads and Magnetic Resonance Metabolic Features. Front Neurosci (2017) 11:398. doi: 10.3389/ fnins.2017.00398 
51. Taschler B, Ge T, Bendfeldt K, Müller-Lenke N, Johnson TD, Nichols TE. Spatial Modeling of Multiple Sclerosis for Disease Subtype Prediction. Med Image Comput Comput Assist Interv (2014) 17(Pt 2):797-804. doi: 10.1007/ 978-3-319-10470-6_99

52. Mato-Abad V, Labiano-Fontcuberta A, Rodríguez-Yáñez S, García-Vázquez R, Munteanu CR, Andrade-Garda J, et al. Classification of Radiologically Isolated Syndrome and Clinically Isolated Syndrome With Machine-Learning Techniques. Eur J Neurol (2019) 26(7):1000-5. doi: 10.1111/ene.13923

53. Kim H, Lee Y, Kim YH, Lim YM, Lee JS, Woo J, et al. Deep Learning-Based Method to Differentiate Neuromyelitis Optica Spectrum Disorder From Multiple Sclerosis. Front Neurol (2020) 11:599042. doi: 10.3389/ fneur.2020.599042

54. Wang Z, Yu Z, Wang Y, Zhang H, Luo Y, Shi L, et al. 3D Compressed Convolutional Neural Network Differentiates Neuromyelitis Optical Spectrum Disorders From Multiple Sclerosis Using Automated White Matter Hyperintensities Segmentations. Front Physiol (2020) 11:612928. doi: 10.3389/fphys.2020.612928

55. Kamińska J, Koper OM, Piechal K, Kemona H. Multiple Sclerosis - Etiology and Diagnostic Potential. Postepy Hig Med Dosw (Online) (2017) 71(0):55163. doi: 10.5604/01.3001.0010.3836

56. Zurita M, Montalba C, Labbé T, Cruz JP, Dalboni da Rocha J, Tejos C, et al. Characterization of Relapsing-Remitting Multiple Sclerosis Patients Using Support Vector Machine Classifications of Functional and Diffusion MRI Data. NeuroImage Clin (2018) 20:724-30. doi: 10.1016/j.nicl.2018.09.002

57. Barnett Y, Garber JY, Barnett MH. MRI Biomarkers of Disease Progression in Multiple Sclerosis: Old Dog, New Tricks? Quant Imaging Med Surg (2020) 10 (2):527-32. doi: 10.21037/qims.2020.01.04
58. Pinto MF, Oliveira H, Batista S, Cruz L, Pinto M, Correia I, et al. Prediction of Disease Progression and Outcomes in Multiple Sclerosis With Machine Learning. Sci Rep (2020) 10(1):21038. doi: 10.1038/s41598-020-78212-6

59. Zeng C, Gu L, Liu Z, Zhao S. Review of Deep Learning Approaches for the Segmentation of Multiple Sclerosis Lesions on Brain MRI. Front Neuroinform (2020) 14:610967. doi: 10.3389/fninf.2020.610967

60. Deng J, Dong W, Socher R, Li L-J, Li K, Fei-Fei L. Imagenet: A Large-Scale Hierarchical Image Database. In: 2009 IEEE Conference on Computer Vision and Pattern Recognition (2009). p. 248-55.

Conflict of Interest: The authors declare that the research was conducted in the absence of any commercial or financial relationships that could be construed as a potential conflict of interest.

Publisher's Note: All claims expressed in this article are solely those of the authors and do not necessarily represent those of their affiliated organizations, or those of the publisher, the editors and the reviewers. Any product that may be evaluated in this article, or claim that may be made by its manufacturer, is not guaranteed or endorsed by the publisher.

Copyright (c) 2021 Moazami, Leferre-Utile, Papaloukas and Soumelis. This is an openaccess article distributed under the terms of the Creative Commons Attribution License (CC BY). The use, distribution or reproduction in other forums is permitted, provided the original author(s) and the copyright owner(s) are credited and that the original publication in this journal is cited, in accordance with accepted academic practice. No use, distribution or reproduction is permitted which does not comply with these terms. 